\title{
GEOVISUALIZACIÓN DE ESCENARIOS DE CAMBIO CLIMÁTICO PARA ANDALUCÍA: DISEÑO DEL GEOVISOR CAMBIA.CLIMASIG.ES
}

\author{
Juan Mariano CAMARILLO NARANJO, José Ignacio ALVAREZ FRANCOSO, \\ Natalia LIMONES RODRÍGUEZ, Mª Fernanda PITA LÓPEZ, \\ Mónica AGUILAR-ALBA \\ Departamento de Geografía Física y Análisis Geográfico Regional,Universidad de Sevilla, España. \\ jmcamarillo@us.es, jalvarez2@us.es, natalialr@us.es, mfpita@us.es, malba@us.es
}

\section{RESUMEN}

Esta comunicación presenta los resultados de la investigación llevada a cabo para el desarrollo de un geovisor diseñado para la explotación y difusión de los datos de escenarios regionales de cambio climático para Andalucía. Este geovisor está basado en la generación de servicios interoperables OGC (Open Geospatial Consortium) de visualización. Este trabajo es fruto de la colaboración entre el Grupo de Climatología del Departamento de Geografía Física de la Universidad de Sevilla y la Consejería de Medio y Ordenación del Territorio (CMAOT) de la Junta de Andalucía.

El conocimiento generado en términos de técnicas de geovisualización de BIG DATA espaciales ha sido transferido a la Red de Información Ambiental de Andalucía (REDIAM), con el objeto de poder ser aplicado a otros ámbitos de la información climatológica y ambiental. Los datos de partida proceden de los escenarios regionalizados para Andalucía a partir de técnicas de downscalling estadístico, a partir de la modelización (3 modelos CNMC3, ECHAM5 y EGMAN) y de los escenarios del IV Informe del IPCC (a1b, a2 y b1). Este trabajo ha supuesto la implementación de una base de datos espacial de gran volumen y difícil manejo que ha requerido el desarrollo de una nueva línea de investigación y desarrollos que permitiera el análisis y tratamiento de los datos acumulados para su explotación y difusión. El resultado de esta colaboración es un geovisor que permite consultar para la región, a una resolución de 200 metros, escenarios de temperatura media y precipitación para tres horizontes temporales (2011-2040, 2041-2070, 2071-2100) combinando modelos, escenarios y sus correspondientes indicadores de cambio.

Palabras clave: geovisualización, cambio climático, escenarios, bases de datos espaciales, BIG DATA espacial, Andalucía, Software libre.

\begin{abstract}
This paper presents the results of research carried out for the development of a GeoVisor designed for exploitation and dissemination of data of regional climate change scenarios for Andalusia. This GeoVisor is based on the generation of interoperable OGC display. This work is the result of collaboration between the Group of Climatology Department of Physical Geography of the University of Seville and the Environment and Spatial Planning Administration of the Andalusian Government.
\end{abstract}


The knowledge generated in terms of techniques geovisualization BIG DATA space has been transferred to the Environmental Information Network of Andalusia (REDIAM), in order to be able to be applied to other areas of the climatological and environmental information. The input data come from the regionalized scenarios for Andalusia from techniques of statistical downscalling, from modeling (3 models CNMC3, ECHAM5 and EGMAN) and scenarios IV Report of the IPCC (A1B, A2 and B1). This work has involved the implementation of a spatial database of high volume and difficult handling has required the development of a new line of research and development that would allow the analysis and processing of data accumulated for exploitation and dissemination. The result of this collaboration is a GeoVisor that provides access to the region, at a resolution of 200 meters, scenarios average temperature and precipitation for three time horizons (2011-2040, 2041-2070, 20712100) combining models, scenarios and corresponding indicators of change.

Key words: geovisualization, climate change scenarios, spatial databases, spatial BIG DATA, Andalusia, Free Software.

\section{INTRODUCCIÓN}

En los momentos actuales se asiste a un aumento exponencial de los datos y de la información climática así como a un aumento paralelo de sus potenciales usuarios, tanto en el seno de la comunidad científica como fuera de ella. El cambio climático es en buena medida responsable de este fenómeno. Su amenaza ha obligado a i) incrementar las investigaciones relativas al funcionamiento del sistema climático actual y futuro y a producir los crecientes datos climáticos que esta tarea requiere; ii) adoptar, por parte de los gobiernos, políticas conducentes a mayores niveles de sostenibilidad climática y hacer el seguimiento de las mismas; iii) adoptar políticas de adaptación a los futuros cambios climáticos por parte de los poderes públicos, las empresas etc., encaminadas a conformar sociedades más resilientes al cambio climático.

Ello ha propiciado la aparición de un nuevo paradigma, denominado por algunos ya como el cuarto paradigma (Edwards et al, 2011), que responde al doble reto que la ciencia climática se plantea actualmente acerca de los datos tendente a una política de datos más abiertos y más accesibles y manejables por parte de usuarios no expertos en climatología. De esta manera el conjunto de la sociedad puede reducir su vulnerabilidad frente a la variabilidad y el cambio climático, aumentando al mismo tiempo su capacidad para aprovechar las oportunidades que los nuevos escenarios climáticos puedan presentar (Overpeck et al, 2011).

La dificultad es especialmente intensa en el caso de los datos derivados de modelos de simulación del clima del futuro, piezas clave para la elaboración de los programas de adaptación. Tales datos son muy abundantes y supuestamente amigables, a pesar de lo cual son muy raramente usados con total corrección y conocimiento por parte de los usuarios no expertos (Edwards, 2010; Tang and Desai, 2012).

En consonancia con estas premisas, desde hace más de una década la Consejería de Medio Ambiente y Ordenación del Territorio (CMAOT), a través de la Red de Información Ambiental de Andalucía (REDIAM), ha venido colaborando con diferentes grupos de investigación en trabajos relacionados con el análisis y evaluación de información ambiental, en especial de la información climática. En este contexto surge el proyecto de elaboración de Escenarios Locales de Cambio Climático de Andalucía 
(ELCCA) actualizados al $4^{\circ}$ Informe del IPCC, como colaboración de la Fundación para la Investigación del Clima y del equipo técnico de la REDIAM en el marco de lo previsto en Programa de Andaluz de Adaptación al Cambio Climático. El objetivo es el desarrollo y elaboración de los escenarios regionales de cambio climático mediante técnicas de downscaling para las temperaturas y precipitaciones que acerquen a científicos, técnicos, políticos, administración y ciudadanos en general, los efectos esperados del Cambio Climático sobre la región andaluza (Rediam, 2014).

Asimismo la CMAOT está haciendo especial hincapié en el uso y la investigación en el área de las nuevas tecnologías de la información para aplicaciones para temáticas específicas, destacando el diseño de servicios de información interoperables y la implementación de visores de información geográfica desarrollados. A partir de la colaboración entre el Departamento de Geografía Física y AGR de la Universidad de Sevilla y la Consejería de Medio Ambiente y Ordenación del Territorio de la Junta de Andalucía se pone en marcha el proyecto "Diseño y producción de servicios de datos climáticos interoperables y soluciones de visualización", con el objetivo de explotar y difusión de los datos generados de escenarios regionales de cambio climático. Así pues, el objetivo del presente trabajo es presentar los resultados de la investigación llevada a cabo por el Grupo de Climatología de la Universidad de Sevilla para diseñar y desarrollar un modelo de base de datos y una herramienta de geo-visualización que permita, mediante el uso de servicios de datos interoperables que han permitido poner en marcha el Sistema CambiA.

\section{FUENTES, DATOS Y METODOLOGÍA}

El acceso y manejo de grandes bases de datos de información climática, debido a la complejidad de los formatos en los que se presentan en la actualidad, restringe su uso a un número muy reducido de usuarios incluso dentro de las esferas científicas o técnicas. Sin embargo, la importancia de contar con herramientas de fácil acceso que permitan el conocimiento del funcionamiento complejo del sistema climático aparece como una de las claves actuales. En este sentido podemos afirmar que la geo-visualización de los datos procedentes de estas grandes bases de datos climáticas globales es una herramienta poderosa y útil para conseguir el objetivo de hacer que estos datos puedan alcanzar a un número cada vez mayor de usuarios.

La investigación climatológica actual, especialmente en la última década, ha asistido a la eclosión de la producción de grandes bases de datos climáticas globales realizadas por diferentes organismos internacionales, cuyo denominador común es la disponibilidad. En este caso, el desafío del diseño de un sistema de visualización es mayor por el hecho de que el aumento de la resolución espacial de la rejilla de datos produce un incremento significativo del volumen de datos, que alcanza ahora el orden de los millares de millón. Los datos empleados de entrada se han llevado a una rejilla de 200 metros de resolución espacial máxima y son los siguientes:

- Datos de precipitación y temperatura media mensual futuras obtenidas a partir de las variables originales calculadas para las proyecciones realizadas para el siglo XXI. Los datos de partida proceden de los escenarios regionalizados para Andalucía a partir de técnicas de downscalling estadístico, a partir de la modelización (3 modelos de circulación general CNMC3, ECHAM5 y EGMAN) y de los escenarios del IV Informe del IPCC (a1b, a 2 y b1). Cada uno de ellos disponible para tres horizontes temporales (2011-2040, 2041-2070, 2071-2100). 
- Indicadores de cambio calculados como las diferencias del modelo medio respecto al periodo de referencia 1961-2000 para los distintos escenarios de emisiones y periodos futuros.

Este tipo de información se engloba en lo que se considera Big Data espacial, datos cuyo volumen, diversidad y complejidad requieren nueva arquitectura, técnicas, algoritmos y análisis para gestionar y extraer valor y conocimiento oculto en ellos. En este caso, la dificultad del diseño de un sistema de visualización de estos grandes volúmenes de datos climáticos es mayor a los habituales por el hecho de que el aumento de la resolución espacial de la rejilla de datos produce un incremento significativo del volumen de datos, que alcanza ahora el orden de los millares de millón. Para ello el sistema se basa en una arquitectura multinivel en tres capas, implementadas con software y componentes de código abierto: una capa de datos, una capa de negocio/ aplicaciones y una capa de presentación/usuario tal como se representa en la figura 1.

Los datos que actualmente muestra el sistema son los resultados, para las variables de precipitación total y temperatura media mensual, del proyecto ELCCA, en formato GeottTIFF, que posteriormente son importados a una Base de Datos espacial relacional PostGIS v.2.0 construida con sistema gestor de bases de datos relacional de código abierto PostgreSQL v. 9.3.4. En la capa de aplicaciones se desplegó un servidor de mapas GeoServer v.2.5 que se comunica con el sistema gestor de la base de datos para obtener las variables y servirlas como servicios interoperables. La capa de presentación o capa de usuario constituye el componente visible del sistema. La interfaz gráfica se ha desarrollado en Openlayers v.2.13 sobre HTML v5 y CSS v3.

Considerando el alto número de registros que se almacenarán en esta base de datos (más de 6 mil millones), y que no deben realizarse actualizaciones en la misma, se ha optado por diseñar un modelo de datos simple, se han aplicado dos técnicas con el fin de optimizar la velocidad de renderizado de las imágenes en pantalla, lo que unido a la implementación de peticiones WMS teseladas, incrementa de manera significativa la velocidad de presentación en pantalla de las capas, mejorando la experiencia del usuario. Se accede al visor a través de la dirección: http://cambia.climasig.es.

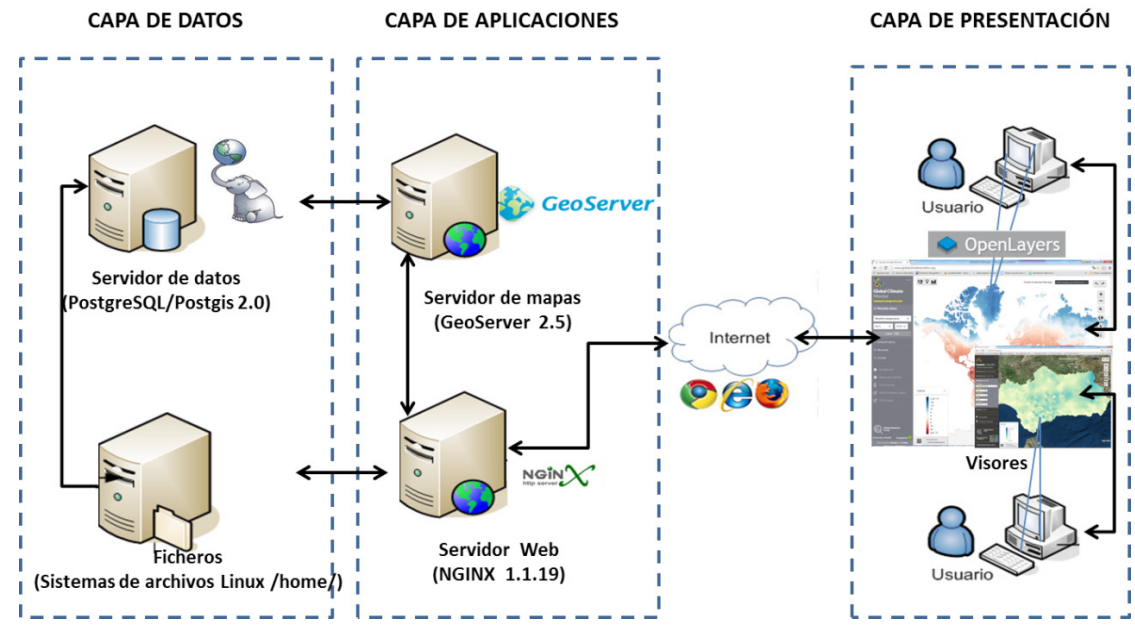

Fig. 1. Arquitectura del sistema CambiA. Fuente: Elaboración propia. 
El servidor cartográfico utiliza el software de código abierto Geoserver v2.5 elegido por dos razones, su diseño orientado a la interoperabilidad y por la facilidad de implementación. Las capas se han publicado en el servidor cartográfico a partir de las consultas espaciales mencionadas en el apartado anterior. Para su difusión se ha habilitado un servicio único WMS (visualización) que contiene todas las capas para todas las fechas posibles.

\section{RESULTADOS}

El geovisor CambiaA permite un conocimiento del clima que responde, a su vez, a las necesidades del aprendizaje para el siglo XXI, que debe enfocarse de manera diferente en el contexto del desarrollo de la sociedad digital y el acceso universal, considerando la diversidad de aplicaciones y usuarios que deben tenerse en cuenta. El progreso y la innovación ya no se ven obstaculizados por la capacidad de recopilar datos, sino por la capacidad de extraer el valor de los datos y convertir datos en conocimiento. El sistema CambiA consigue este objetivo a partir de las posibilidades de las técnicas de geovisualización de BIG DATA espaciales y ha sido transferido a la Red de Información Ambiental de Andalucía (REDIAM), con el objeto de poder ser aplicado a otros ámbitos de la información climatológica y ambiental.

El visor carga servicios interoperables a distintas resoluciones (2000, 1000 y 200 metros dependiendo de la escala de visualización) relativos a las variables climáticas proyectadas y a los indicadores de cambio calculados (Figura 2). Estos servicios se cargan en forma de capas ordenadas en cuatro categorías: variables mensuales, variables anuales, indicadores de cambio mensual e indicadores de cambio anual

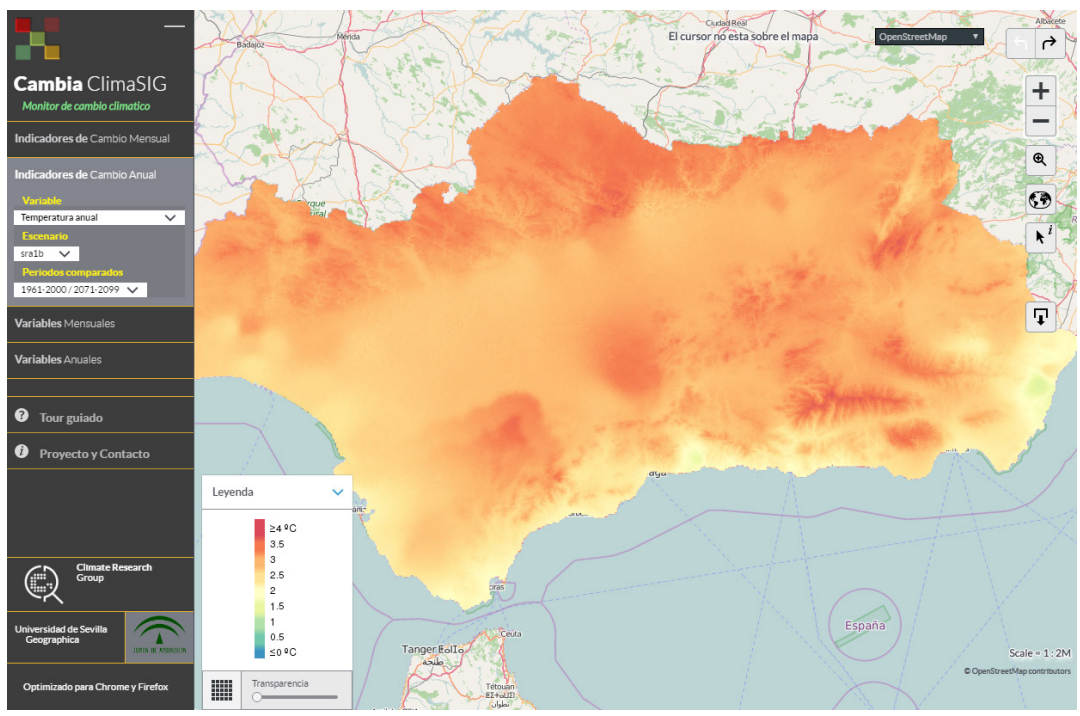

Fig. 2. Interfaz de usuario del visor CambiA. Fuente: http://cambia.climasig.es.

El visor CambiA dispone de un aspecto corporativo y una forma de navegación que hace que el usuario interactúe de una manera más cómoda y rápida con la aplica- 
ción. Una vez que el usuario ejecuta en el navegador la dirección www.cambia.climasig.es, éste despliega una ventana desde donde se puede observar el visor al completo con una serie de capas de información activadas por defecto.

El menú de selección de capas permite al usuario interactuar con la selección de variables climáticas e indicadores de cambio, modelos de circulación generales, escenarios de emisiones, periodos a futuro -treintenios 2011-2040, 2041-2070 y 20712100- y meses. La velocidad de carga y de navegación se obtiene a partir de la generación de vistas sql parametrizadas ejecutadas por el servidor de datos (POstgreSQL/ Postgis), lo que, unido a técnicas de indexado muy eficientes, permite la navegación rápida espacial en el visor, elemento indispensable para que un geovisor cumpla con los requisitos de navegación de los usuarios.

Además, el geovisor presenta funcionalidades de zoom, panning, getfeatureinfo y exportación del mapa. Finalmente el visor permite la carga de distintos servicios de mapas generales para ser utilizados como fondo cartográfico. Pueden ser elegidos entre ocho posibilidades que se ofrecen: Openstreetmap, 4 mapas base de Esri (Basemap, Satellite, Ocen basemap y Shaded relief y Stamen toner-lite). Las coordenadas están expresadas en grados decimales para facilitar la interpretación del usuario. Todas las capas se muestran en la proyección ETRS89 UTM 30.

\section{DISCUSIÓN}

El visor CambiA responde a diversas necesidades actuales de la información y servicios climáticos. La creciente disponibilidad de datos es una tendencia de importancia estratégica que ofrece una gran oportunidad, como forma de reducir la carga de respuesta a los usuarios, posibilidad de generar nuevos productos, reducir el desfase en la producción del dato o permitir el acceso a multitud de usuarios diversos. CambiA cumple el objetivo de avanzar en las oportunidades del Big Data en la difusión de la información climática en Andalucía cumpliendo así el Artículo 12 del Programa de Comunicación del Anteproyecto de Ley Andaluza de Cambio Climático (CMAOT, 2014), cuyo objeto es fomentar la participación activa de la sociedad en la lucha contra el cambio climático que incluye en su apartado a) Acciones de sensibilización y educación ambiental para la mejora del conocimiento sobre cambio climático en Andalucía y d) Herramientas y procedimientos para el acceso del público a la información sobre cambio climático y sus efectos.

Por otro lado, en relación a los servicios climáticos entendidos como toda "información sobre el clima" haciendo referencia a los datos, información, conocimiento y experiencia para comprender y configurar la adaptación, la mitigación, los impactos, la vulnerabilidad y evaluación de los riesgos asociados, cada vez existen más sectores y particulares interesados que demandan información sobre los cambios, las consecuencias, las probabilidades y la gama de posibles resultados relacionados con el clima. Paralelamente, este proyecto se inserta dentro del paradigma de open science, particularmente en su esfera de ciencia para la ciudadanía-citizen science-, en el sentido de generar herramientas que permitan que datos científicamente complejos sean más accesibles, entendibles y manipulables al potencial de usuarios ciudadanos o colectivos de la información relativa a la ciencia en general y, en este caso, a la ciencia del cambio climático en particular. 


\section{AGRADECIMIENTOS}

Red de Información Ambiental de Andalucía (REDIAM) y Consejería de Medio Ambiente y Ordenación del Territorio (CMAOT)

\section{REFERENCIAS}

Consejería de Medio Ambiente y Ordenación del Territorio de la Junta de Andalucía (2014). Anteproyecto de Ley Andaluza de Cambio Climático. Recuperado de http://www.juntadeandalucia.es/medioambiente/PAC/Noticias/Imagenes/ anteproyecto ley cambio $\% 20$ climatico.pdf?lr=lang_es

Edwards, P.N. (2010). A Vast Machine: Computer Models, Climate Data, and the Politics of Global Warming, MIT Press, $552 \mathrm{pp}$.

Edwards, P. N., M. S. Mayernik, A. L. Batcheller, G. C. Bowker, and C. L. Borgman (2011). Science Friction: Data, Metadata, and Collaboration, Social Studies of Science, 41:5 667-690. DOI: 10.1177/0306312711413314

Overpeck, J.T., Meehl, G.A., Bony, S and Easterling, D.R. (2011). Climate data challenges in the $21^{\text {st }}$ century, Science, vol. 331, 700-702. DOI: 10.1126/ science. 1197869

Rediam (2014). El Clima de Andalucía en el siglo XXI. Escenarios Locales de Cambio Climático de Andalucía. Actualización al $4^{\circ}$ Informe del IPCC. Red de Información Ambiental de Andalucía, Consejería de Medio Ambiente y Ordenación del Territorio de la Junta de Andalucía. Recuperado de http://www. juntadeandalucia.es/medioambiente/portal_web/rediam/documentos/docs/clima/ clima_andalucia_siglo_XXI_ELCC_IPCC $\overline{4}$.pdf

Tang, S., and S. Desai (2012). Usable science? The UK Climate Projections 2009 and decision support for adaptation planning, Weather Climate and Society, vol. 4, 300-313. DOI: http://dx.doi.org/10.1175/WCAS-D-12-00028.1. 